\title{
Survey of Beamed Energy Propulsion Concepts by the MSFC Space Environmental Effects Team
}

\author{
P.A. Gray" . M.K. Nehls", D.L. Edwards", M.R. Carruth, Jr". \\ ${ }^{*}$ ICRC Huntsville, AL 35816 \\ "ED3I Environmental Effects Group \\ Marshall Space Flight Center, AL 35812
}

\begin{abstract}
This will be a survey paper of work that was performed by the Space Environmental Effects Team at NASA's Marshall Space Flight Center in the area of laser energy propulsion concepts. Two types of laser energy propulsion techniques were investigated. The first was ablative propulsion, which used a pulsed ruby laser impacting on single layer coatings and films. The purpose of this investigation was to determine the laser power density that produced an optimum coupling coefficient for each type of material tested. A commercial off-the-shelf multi-layer film was also investigated for possible applications in ablative micro-thrusters, and its optimum coupling coefficient was determined. The second type of study measured the purely photonic force provided by a $300 \mathrm{~W}$ CW YAG laser. In initial studies, the photon force resulting from the momentum of incident photons was measured directly using a vacuum compatible microbalance and these results were compared to theory. Follow-on work used the same CW laser to excite a stable optical cavity for the purpose of amplifying the available force from incident photons.
\end{abstract}

\section{INTRODUCTION}

There are two techniques for using laser photons to produce propulsive forces that will be discussed in this paper. One technique is laser ablation, which uses short, intense pulses of laser light energy to ablate a small amount of material. The laser has a very short pulse in the nano to picosecond range. This provides the instantaneous power necessary to ablate the surface of a fuel source but not cause damage to the spacecraft. This technique can also be applied to spacecraft that have an on board laser source. Typically ground based laser systems have been considered for de-orbiting debris 
particles from low earth orbit. On board lasers have also been considered for ablative micro-thruster applications, Ref Claud Phipps here. The other technique takes advantage of the momentum imparted to a surface when photons strike the surface of a material. This technique is applicable to laser sailing using a laser as a manmade source of photons. The laser has the advantage that large amounts of energy can be concentrated in a small area, thus reducing the size and mass of the sail.

\section{ABLATIVE LASER INTERACTION WITH MATERIAL}

\section{BACKGROUND}

The Space Environmental Effects (SEE) team, at Marshall Space Flight Center, began working in the area of laser interaction with materials by experimentally investigating ablative forces generated when lasers interact with material. The SEE team designed a torsion balance and used a pulsed ruby laser to characterize the relationship between laser power density and ablative force for various materials representative of orbital debris. A natural transition of the SEE team was to utilize their experience, with lasermaterial interaction, and the existing test capability to investigate beamed energy propulsion. The most recent work, for the SEE team is in the area of multi-layered thin films in a micro-thruster application. The concentration for the SEE team will be focused on optimizing the thin film composition for micro-thruster applications.

\section{APPARATUS}

The laser used to produce the ablative force is a Lumonics QSR3, 3J pulsed ruby laser $(694 \mathrm{~nm})$ with a Q-switch to create a narrow pulse. This laser can produce energies as high as $5 \mathrm{~J}$ with a 25 nanosecond (ns) pulse width. The torsion balance is located in a vacuum chamber operating in the $1 \times 10^{-7}$ torr range. The chamber consists of a 13-inch conflat cross with a window at the top to view the pendulum and a window at the side to illuminate the pendulum for recording on videotape. The laser enters the chamber through an anti-reflection (AR) coated window that is optimized for $694 \mathrm{~nm}$. The oscillation of the torsion balance is recorded using a video recorder. The positive to negative displacement $2 \theta_{\max }$, and the period of the pendulum are measured post test by replaying the videotape. The laser pulse is captured with a Molectron Model P5-01 laser pulse detector connected to a Tektronics model TDS460A, $500 \mathrm{MHz}$, digital storage oscilloscope. The pulse width is taken at full width at half maximum (FWHM). The total energy per pulse is measured using a Molectron Model EPM1000 pulsed laser energy meter with a model J25LP-RUBY energy detector. The energy detector samples $8.5 \%$ of the beam using a quartz beam splitter. The pulse detector was taken out of its case and mounted so that it detected the diffuse reflection off of the ceramic diffuser on the energy detector. This eliminated the need for a secondary beam splitter for the pulse detector. The laser energy is attenuated by using a beam splitter stack and 0.4 and 0.8 neutral density (ND) filters. The $1 / \mathrm{e}^{2}$ beam diameter is measured with a Spiricon model LBA-300PC 8 -bit beam profiler. The beam diameter is varied using different focal length lenses 
with the sample located at the focal length of each lens. Two lenses were used for these tests, a $350 \mathrm{~mm}$ and a $500 \mathrm{~mm}$. The $500-\mathrm{mm}$ lens produces a $0.41 \mathrm{~mm}$ dia. spot size and the $350-\mathrm{mm}$ lens produces a $0.3 \mathrm{~mm}$ dia. spot size.

\section{CALCULATIONS}

The coupling coefficient is the ratio of the momentum transferred to the pendulum to the laser power density. It is a measure of the efficiency of converting light energy to kinetic energy through the process of ablation. It is plotted as a function of the laser power. Power is defined as the laser energy divided by the pulse width and the beam area, which gives the familiar $W / \mathrm{cm}^{2}$. On a plot of coupling coefficient versus laser power, the optimum coupling coefficient occurs at the highest point on the curve. The laser power density corresponding to the optimum coupling coefficient is the point of maximum efficiency in converting the laser energy to momentum. Increasing the power further will produce more momentum but the efficiency decreases. Laser power levels below that which corresponds to the optimum coupling coefficient produce force at less efficiency. In addition, the force produced drops off at a very high rate as laser power is decreased below that corresponding to the optimum coupling coefficient. Power levels should be equal to or above the optimum coupling coefficient in order to insure that enough ablation occurs to produce a force capable of significantly changing the velocity of a debris particle or for use as a propulsive force.

A simple torsion balance was used for measuring the momentum produced by the ablation process. The balance consists of a thin metal wire with a rotating pendulum suspended in the middle. The wire is fixed at both ends. Figure 1.0 defines the radius and angular displacement parameters for the torsion balance. Wire, of a known torsion spring constant, allows calculation of the maximum force or thrust produced by the ablation. The period of the pendulum is also measured. The momentum produced by the ablation is the product of the average force and the portion of the period from the zero position to the maximum displacement position, i.e. maximum spring force or zero velocity. Since the force varies as a function of angular displacement, the force is zero at zero displacement and a maximum at the maximum angular displacement $\theta_{\max }$. The average force over the time interval from zero to $\theta_{\max }$ is simply the maximum force divided by 2 .

$$
\mathrm{F}_{\mathrm{ave}}=\mathrm{C} \theta_{\max } / 2 \mathrm{r}
$$

where $\mathrm{C}$ is the torsion spring constant, $\mathrm{r}$ is the distance from the center of the laser impact sight to the center of the tungsten wire, and theta is the angle of displacement in radians. 
The time interval from zero to max displacement is simply $1 / 4$ the period $T$. So the momentum

$$
\mathrm{M}=\mathrm{F}_{\mathrm{ave}} \Delta \mathrm{T}
$$

where $\Delta \mathrm{T}=1 / 4 \mathrm{~T}$.
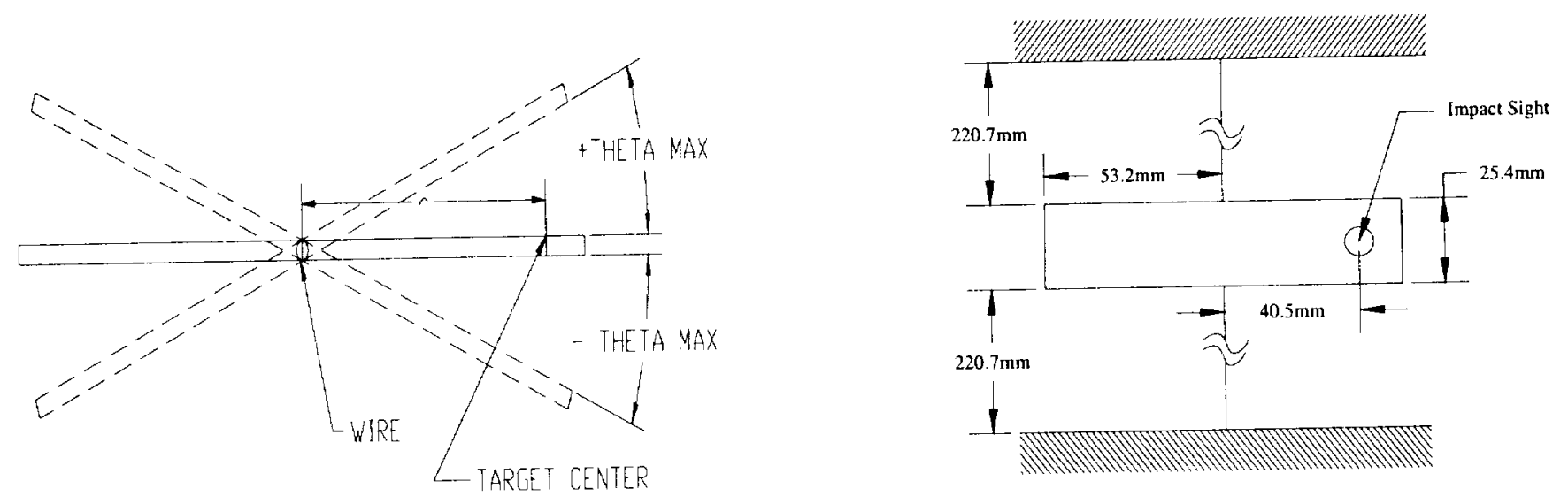

Figure 1.0 Schematics of torsion pendulum

\section{RESULTS}

The ablative coupling coefficients for several coatings and materials were measured. A graph of the coupling coefficient curves in located in Figure 2.0. Also a muli-layer material, Silver - Teflon was measured and compared to bare aluminum without the Teflon outer layer. The Sliver-Teflon material had an order of magnitude increase in coupling coefficient at the expense of a much larger damaged region. The outer Teflon layer served to contain the gases evolved during the ablation process. The Teflon later formed a bubble containing the expanding gases and subsequently ruptured forming a crude nozzle which directed the these high pressure gases in a direction normal to the surface of the material. It is thought that the pressure resulting from containing the ablation products and the formation of the nozzle are responsible for the increase in the measured force. If this discovery is to be more than of academic interest as a propulsion technique the amount of material used in each laser hit must be considered. A diameter, two or three times that of the laser beam, was damaged. Mulitilayer materials have been considered for use in ablative micro-thrusters REF Claud Phipps. His technique used a clear material with the ablative fuel on the side away from the laser. The laser passed through the clear layer and ablates the materials coated in the other side. Once the laser has hit an area it is moved across the width of the tape until the entire width of the tape is used. Once the width of the material is consumed it is translated and a fresh area of tape is exposed to translate the laser over in a process analogous to a typewriter ribbon. 


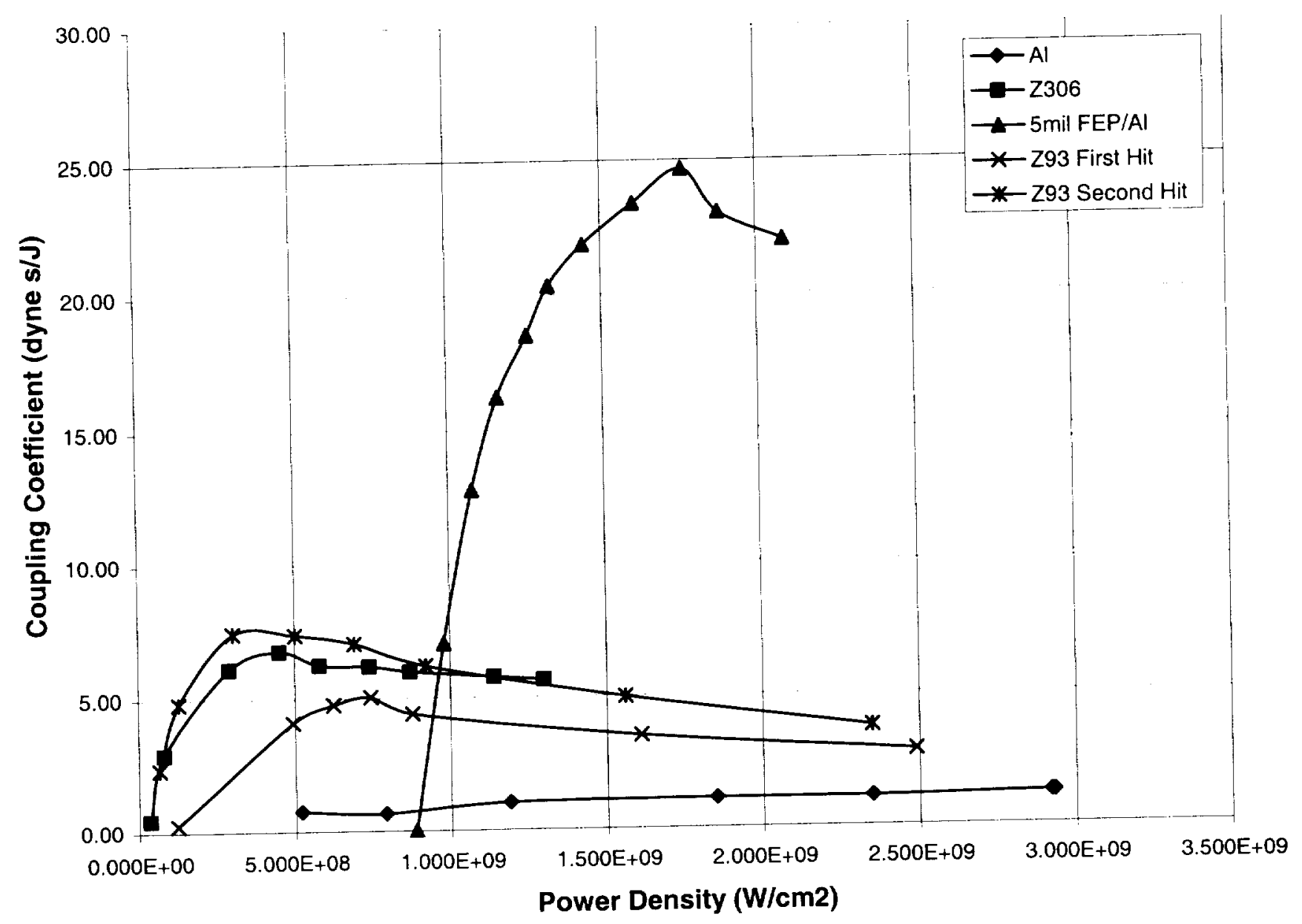

Figure 2.0 Coupling coefficient curves for various materials.

\section{NON-ABLATIVE LASER INTERACTION WITH MATERIAL}

\section{BACKGROUND}

The SEE team has developed the capability to measure photon pressure. This capability was developed to experimentally verify the photon pressure theory and to provide solar sail designers the experimental validation required for mission scenarios. Originally, this photon pressure measurement capability was developed using a full spectrum photon source. The SEE team has expanded the application of this measurement capability to measure the photon pressure from a Continuous Wave (CW) laser source. This pressure measurement capability was expanded to experimentally investigate the amplification of photon pressure. This amplification process uses a semi-stable optical cavity to reflect laser photons back onto the primary photon-driven propulsion system, such as a laser sail. 


\section{APPARATUS}

The test chamber for this experiment consists of a 6 way vacuum cross mounted on an ion pump. The laser beam passes through a $-250 \mathrm{~mm}$ focal length expanding lens then through a standard glass view port. The divergence angle was less than $3^{\circ}$. Since the cosine of this angle is approximately one, the effect of angle was neglected. After it passes through the view port, a $45^{\circ}$ turning mirror directs the expanded laser beam to the target.

The laser being used for this experiment is a Quantronix Model 118,300W peak output Nd YAG CW laser. See the Figure 3.0 for the optical setup for the photon force measurements and Figure 4.0 for the photon amplification setup.

The force-measuring device is a CAHN model D-101 vacuum compatible microbalance. The sample is suspended from the balance with a long Nichrome wire.. When the laser is directed onto the target and the photons impinge on it, they provide an upward force. This is measured by the microbalance as a decrease in target weight. The samples consisted of a thin stainless steel plate in the case of the photon force measurements and a 2" diameter mirror in the case of the amplified photon measurements. The thin plate was chosen because its lightweight and high damage threshold.

Laser power measurements were obtained, using a Molectron Model PM300F-50. The power meter was located after the expanding lens and before the chamber window.

The experimental setup for the photon amplifier consists of an optical cavity. This consists of two $100 \mathrm{~mm}$ focal length (FL) concave mirrors. One of the mirrors (M1) has a small hole drilled in the center for the laser beam to pass through. The mirrors are mounted horizontally facing each other, one above the other, as shown in the schematic in Figure 2. The mirror with the hole in it (M1) is placed at the bottom of the cavity. The top mirror (M2) is suspended directly above the mirror with the hole (M1). The laser beam will enter the cavity by reflecting off a $45^{\circ}$ dielectric mirror.

As a comparison to the two-mirror system which provided an amplified photon force, the system was also run with the lower mirror removed. In this case incoming photons impinged on the mirror only one time. 
SCHEMATIC QIF TEST SETUP

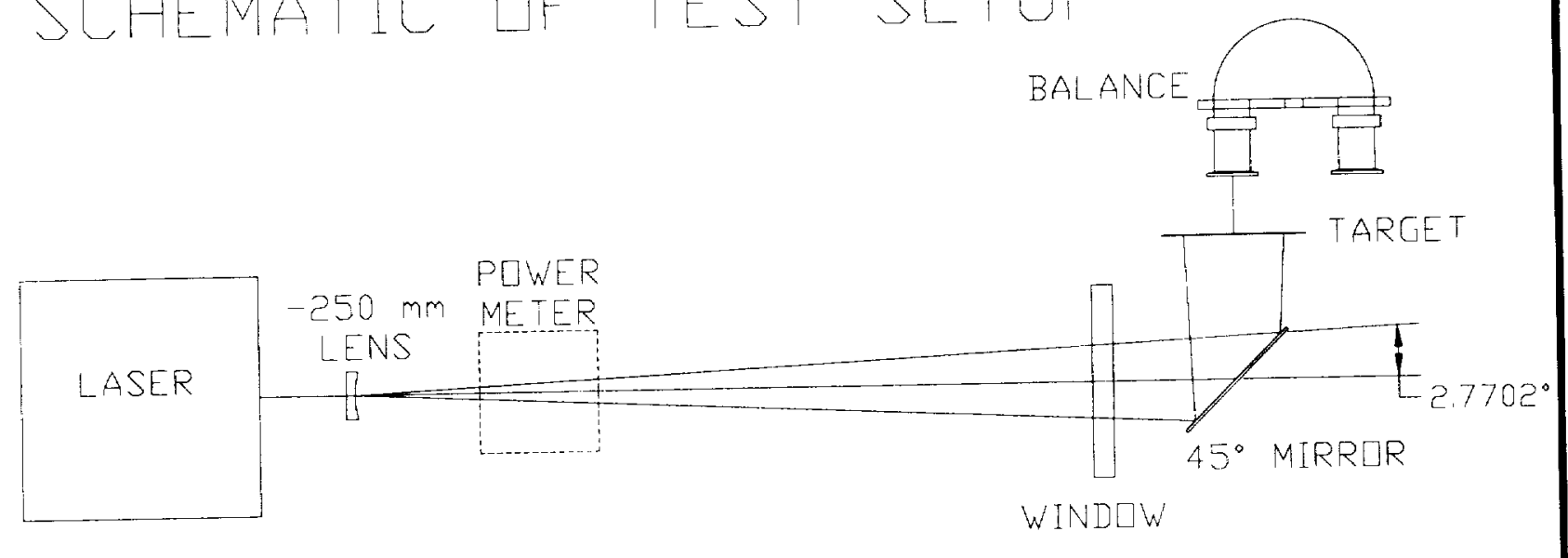

Figure 3.0 Photon force measurement optical setup.

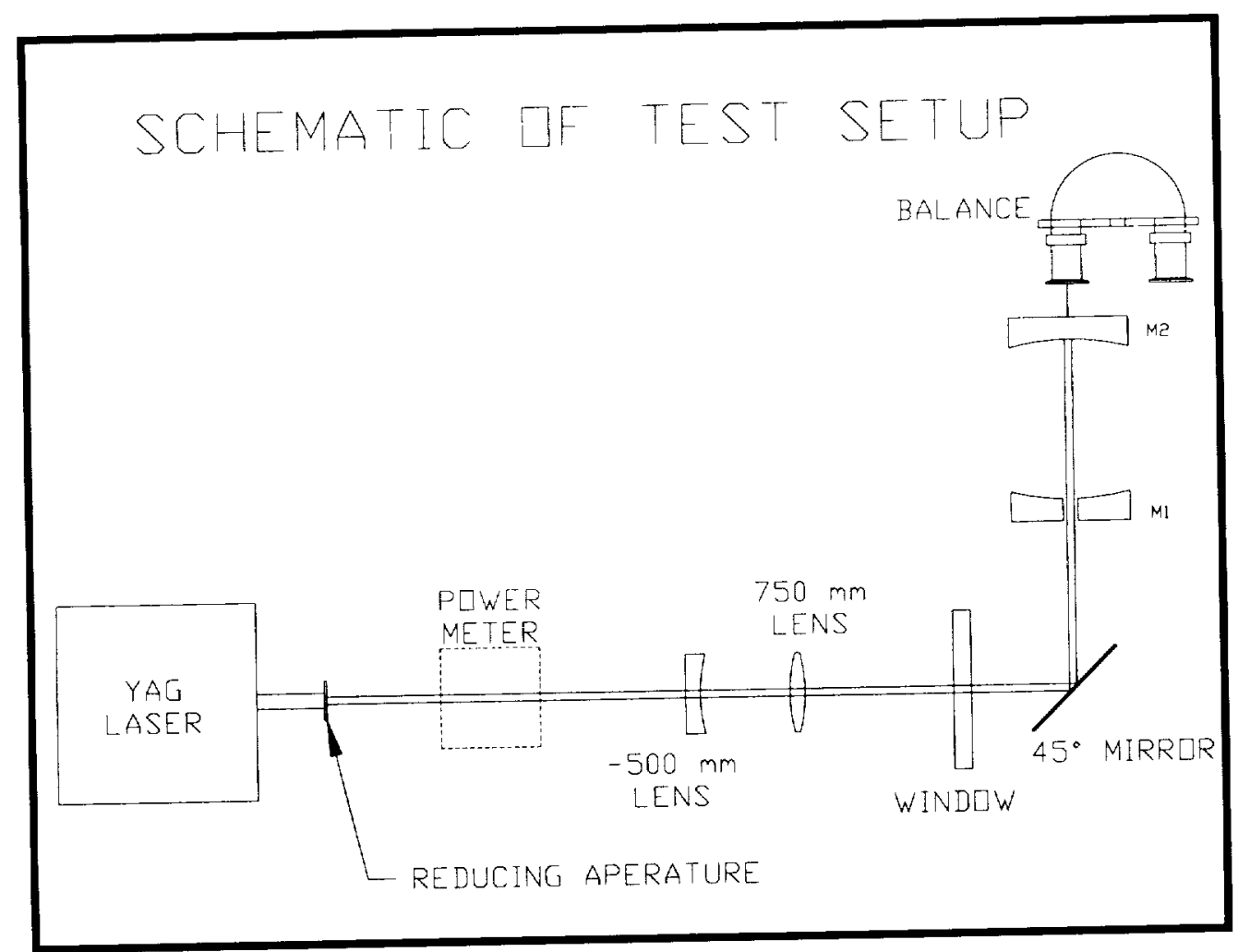

Figure 4.0 Photon amplification optical setup. 


\section{CALCULATIONS}

Working formulas will be presented in this paper. See ref 1 for a detailed derivation of the formulas. The general equation for photon force is:

$$
\mathrm{F}=\mathrm{I} / \mathrm{c},
$$

where $I$ is the total power from the laser in Watts.

For a reflective surface, there is also a momentum contribution due to the reflected photons. A general equation for the photon force on a reflective surface is given by:

$$
\mathrm{F}_{\text {total }}=(\mathrm{V} / \mathrm{c})(1+\mathrm{R})
$$

where $\mathrm{R}$ is the reflectance of the target material at laser wavelength of $1064 \mathrm{~nm}$. For the stainless steel plate $R=0.79$.

A general equation that includes optical component losses is given by:

$$
\mathrm{F}_{\mathrm{calc}}=(\mathrm{I} / \mathrm{c})(1+\mathrm{R})(\eta)
$$

where $\eta$ is an empirical term that takes into account measured losses through optical components. For the measurements of photon force with the stainless steel target $\eta=$ 0.868 .

Now consider multiple photon reflections onto the mirror. The result is that $F_{\text {calc }}$ doubles with each reflection off the second mirror (sail). However, the losses increase with the mirror reflectance squared since each reflection cycle requires two reflections to complete. A general equation for total force that takes into account the loses dues to multiple mirror reflections is:

$$
F_{c a l c}=(\mathrm{Vc})(1+\mathrm{R}) \Sigma\left(\mathrm{R}^{2}\right)^{(\mathrm{N}-1)},
$$

where $\mathrm{N}=$ number of reflection cycles.

A graph of this equation for three different mirror reflectances is found in Figure 1.

In order for equation (9) to be valid, the cavity must be stable. The relationship for a stable cavity is ${ }^{3}$ :

$$
(1-d / 2 f)^{2}<=1,
$$


where $d$ is the distance between the two mirrors and $f$ is the focal length of the mirrors. The assumption is made that both mirrors have the same focal length for this particular argument.

This stability criterion limits the distance between the two mirrors. The separation distance must be less than or equal to 4 times the mirror focal length. The stability criteria is then:

$$
\mathrm{d}<=4 \mathrm{f} \text {. }
$$

\section{RESULTS}

The laser was passed through an expansion lens to reduce the energy density. The lens focal length was $-250 \mathrm{~mm}$ to make the divergence angle as small as possible to reduce off axis losses. The laser power was measured after the diverging lens and before the vacuum chamber window. As a measure of the validity of the photon force measurements, the measured force $\left(\mathrm{F}_{\mathrm{m}}\right)$ was divided by that predicted from laser power measurements $\left(F_{\text {calc }}\right)$. $F_{\text {calc }}$ was calculated using the laser power measurements and equation (9). Since both numbers have error associated with them, a combined error for the ratio, of $4.1 \%$, was calculated using the technique of the propagation of errors. The value of the ratio was $\mathrm{F}_{\mathrm{m}} / \mathrm{F}_{\text {calc }}=0.986$ or $98.6 \%$. Power measurements were taken periodically to compensate for power drift in the laser intensity.

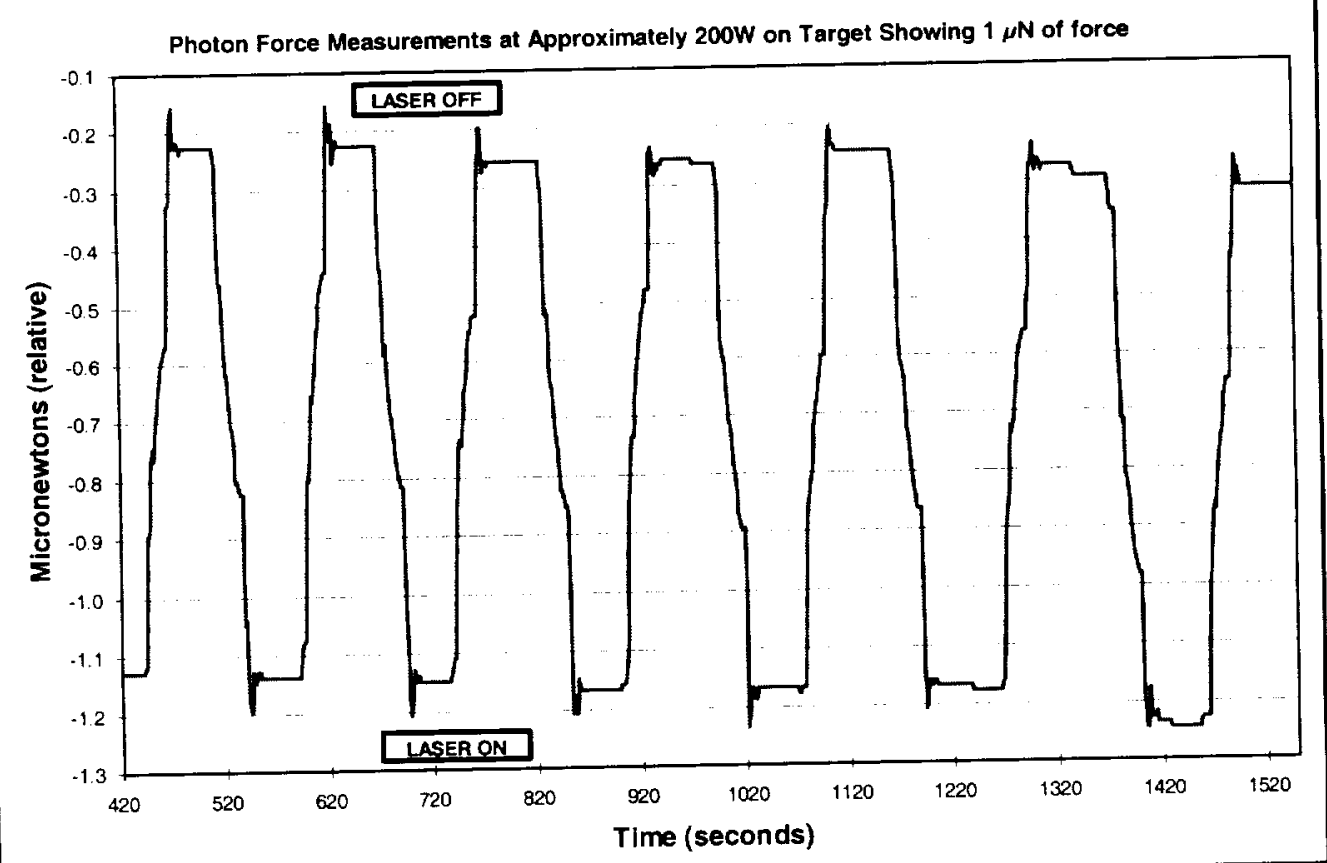

Figure 5.0 Laser force data 
After bread boarding determined that a stable laser cavity was possible with the two $100 \mathrm{~mm}$ FL mirrors, actual amplified force measurements were made in vacuum using the $300 \mathrm{~W}$ Nd YAG CW laser.

An aperture to was used to reduce the beam diameter of the laser to approximately $9.5 \mathrm{~mm}$ diameter from $19 \mathrm{~mm}$. This allowed the beam to pass through the bottom mirror that had a $9.5 \mathrm{~mm}$ diameter hole in it. A $-500 \mathrm{~mm}$ and $750 \mathrm{~mm}$ lens were used in series to adjust the beam diameter slightly and also provide some beam steering so that the beam could be guided through the $9.5 \mathrm{~mm}$ diameter hole. This method did not harm the top mirror but the first mirror focused the beam on to the second mirror causing it to burn. This severely limited the amplification of photons within the cavity. Since alignment was difficult inside the vacuum chamber and space was limited, it is not known at this time if the low amplification number was due to the mirror failure or the beam leaving the cavity through the $9.5 \mathrm{~mm}$ diameter hole in the lower mirror.

For the purposes of comparison the geometry was retained but the bottom mirror was removed. The laser power was increased to produce approximately the same force without the aid of the amplification. One data set at this power level was obtained before the top mirror burned. The measured force was $89 \%$ of the calculated force, which was based on power measurements and system losses. Prior studies have shown measured and calculated photon force to be within a few percent ${ }^{4}$. It is possible that, the mirror degraded prior to failing resulting in the large disparity between calculated and measured force.

The amplification factor (AF) for each experiment is defined by the ratio of measured to calculated force.

$$
\mathrm{AF}=\mathrm{F}_{\mathrm{m}} / \mathrm{F}_{\mathrm{calc}}
$$

The calculated force, $F_{\text {calc }}$, was calculated assuming no amplification in all experiments. An amplification ratio of greater than one indicates that force amplification did occur during the experiment. An average amplification factor for the two amplified force measurements yielded an amplification factor of 2.64.

\section{SUMMARY}

The measurements indicate that the force from laser photons can be accurately measured with a vacuum compatible microbalance. This technique provided repeatable and reliable laser photon force data. Future work will involve the use of actual sail materials. With the current setup the laser energy density is too intense for use on thin polymeric materials. Two factors that will improve this problem are increasing the beam diameter by the use of a larger vacuum chamber and sample, and changing the distribution of energy in the beam from a cone shape to a top-hat shape. Both of these techniques will reduce target hotspots. 
This photon amplification experiment has shown that photon reflections within a stable cavity provided a means of optimizing laser photons. An amplification factor of 2.64 was achieved. The theoretical number for this amplification was not achieved due to time and equipment constraints but the concept has been demonstrated. In future experiments, a larger vacuum chamber and better dielectric mirrors should improve results considerably approaching the amplification factors on the order of 50 . Mirror reflectance is the driver for determining the theoretical maximum amplification factor. The higher the reflectance of the mirrors the more bounces can be obtained before reflective losses reduce photonic forces to unusable levels. 


\section{REFERENCES}

1. P. A. Gray, D.L. Edwards, M. R. Carruth, Jr., Preliminary Photon Pressure Measurements Using a Solar Simulator, AIAA Conference Reno Nevada, January 811, 2001, \#2001-1136.

2.

P. R. Bevington, Data Reduction and Error Analysis for the Physical Sciences, McGraw-Hill, 1969. 